\title{
A Computer Vision Method for Respiratory Monitoring in Intensive Care Environment Using RGB-D Cameras
}

\author{
Haythem Rehouma ${ }^{1}$, Rita Noumeir ${ }^{1}$, Philippe Jouvet ${ }^{2}$, Wassim Bouachir ${ }^{3}$ and Sandrine Essouri ${ }^{2}$ \\ ${ }^{1}$ Ecole de technologie supérieure, Montréal (QC), Canada \\ e-mail: haythem.rehouma.1@ens.etsmtl.ca, rita.noumeir@etsmtl.ca \\ ${ }^{2}$ Pediatric Intensive Care Unit, Sainte-Justine Children's Hospital, Montréal (QC), Canada \\ e-mail: philippe.jouvet@umontreal.ca, sandrine.essouri.hsj@ssss.gouv.qc.ca \\ ${ }^{3}$ LICEF research center, TÉLUQ University, Montréal (QC), Canada \\ e-mail: wassim.bouachir@ teluq.ca
}

\begin{abstract}
This paper presents a novel computer vision method to measure the breathing pattern in intensive care environment. The proposed system uses depth information captured by two RGB-D cameras in order to reconstruct a 3D surface of a patient's torso with a high spatial coverage. The optimal positioning for the sensors is a key step to perform an accurate 3D reconstruction without interfering with patient care. In this context, our hardware setup meets the clinical requirements while allowing accurate estimation of respiratory parameters including respiratory rate, tidal volume and inspiratory time. Our system provides the motion information not only for the top of the torso surface but also for its both lateral sides. Our method was tested in an environment designed for critically ill children, where it was compared to the gold standard method currently used in intensive care units. The performed experiments yielded high accuracy and showed significant agreement with gold standard method.
\end{abstract}

Keywords - Intensive care unit, RGB-D camera, Point cloud, Surface reconstruction, Tidal volume, Respiratory monitoring.

\section{INTRODUCTION}

A Pediatric Intensive Care Unit (PICU) is a special area of a hospital providing intensive care services for the age group categories ranging between birth and 18 years. In an intensive care room, the patient needs intensive and highly accurate control, and is closely monitored on a $24 \mathrm{~h} / 24 \mathrm{~h}$ basis. Any occupied space in the PICU room should not disturb caregivers' work or cause distractions that could hurt patients. For this reason, caregivers need plenty of space around the patient. The intensive care main concern is to monitor the patient's vital signs to ensure his safety and be able to intervene in case of sudden worsening. Among these vital signs, accurate respiration monitoring is crucial. Respiration parameters including respiratory rate (RR), tidal volume (Vt), and retraction signs of the breast (RSB) are collected through clinical examination and several medical devices. If the child is mechanically ventilated $\mathrm{RR}$ and $\mathrm{Vt}$ are directly measured by the ventilator. In spontaneous breathing patients, RR is monitored using thoracic electrodes (plethysmography) that are sensitive to movements with a high rate of erroneous RR measurement. Vt is usually not assessed by medical devices during spontaneous breathing, while RSB are only estimated by clinical examination and are a subjective assessment even when caregivers use a scoring system (Wood score for example [1]).

Recently, there has been interesting research using non-contact devices for tracking and evaluating respiratory activity. For example, Ying-Wen Bai et al. [2] described a temporal differencing method to monitor the breath of the patient in the dark without any contact with the body. This technique is based on the detection of moving objects by image processing. It finds the differences between two continuous $2 \mathrm{D}$ frames to obtain any change of the volume. The main drawbacks of this method are the limited spatial coverage and the high computational time. Xia and Siochi [3] proposed a respiratory monitoring system using a structured-light 3D scanner. This work used only depth images captured by the scanner to calculate the average depth over a thoraco-abdominal zone. This zone was manually determined by placing a translation surface on the thorax in the center of the image. Although this study was implemented in realtime, it was a simple proof of concept using raw depth data to visualize respiratory motion signals. Moreover, the proposed system is not able to determine breathing parameters, such as the respiratory rate and the tidal volume. Tahavori et al. [4] placed an RGB-D scanner above the participant's body to calculate the average depth value of 16 regions of interest on the chest and abdomen to analyze their motion. They applied the principal component analysis to the depth information of these regions and demonstrated that only the first principal component describes nearly $70 \%$ of the motion data variance in chest and abdomen surfaces. Other studies [5, 6] presented a sleep monitoring system using a structured light sensor, where thorax movements are detected by recording the depth information during sleep. However, these works have been shown not suited to the clinical environment and specifically to the intensive care environment.

Our work aims to develop a breathing measurement system within the constraints imposed by the specific nature of intensive care. Using RGB-D sensors [7, 8, 9, 10], our system first aligns point clouds captured from different view angles in a common coordinate system. In a second step, we perform a surface reconstruction from a segmented point cloud region using Poisson method [11]. The surface is subdivided using a hierarchical data structure technique for representing a 3D object, called octree data-structure [12]. The volume is then calculated based on the octree subdivision technique. Finally, we determine $\mathrm{Vt}$ and $\mathrm{RR}$ by analyzing the changes in the calculated volumes.

The rest of the paper is presented as follows: section 2 presents the proposed workflow. In section 3 , we describe the experiments and discuss the obtained results. Finally, section 4 concludes the paper.

\section{THE PROPOSED METHOD}

Our objective is to calculate the volume at each frame and estimate a volume time-curve from the calculated volumes. The detection of change in volume is the key to estimate the volume-time curve. Indeed, Vt and RR can be estimated from these volume-time curves generated by our imaging method. The workflow of the system is given in Fig. 1 and Fig.3. The system uses two calibrated cameras to capture scenes from two viewpoints simultaneously and automatically merge them. Each camera is connected to a separate computer. Each of those computers is connected to a server administrating the two cameras. 


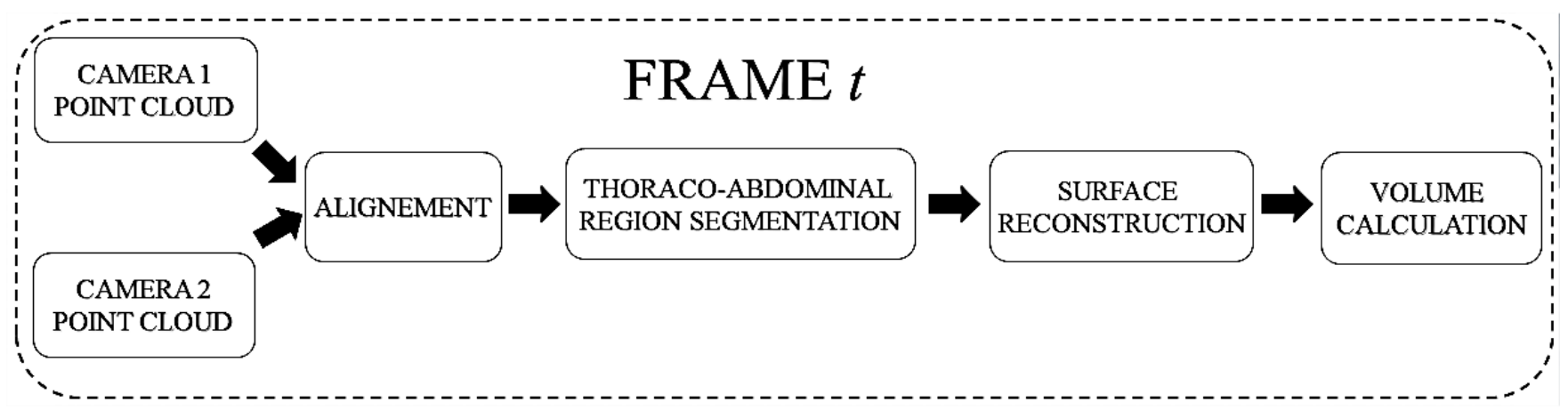

Fig. 1. Volume calculation workflow at frame $t$.

\section{A. Point cloud alignment}

In a point cloud, points are defined by $\mathrm{X}, \mathrm{Y}$, and $\mathrm{Z}$ coordinates. They are intended to represent the external surface of an object. Point clouds are generated from the depth information using the camera SDK. Our objective is to align the point clouds, recorded from different view angles in the same coordinate system in order to perform 3D surface reconstruction with high spatial coverage of the region of interest (ROI). A 2D marker used to align automatically the point clouds is placed on the patient bed. In Fig. 2, each camera infers its relative poses from the detected marker. To achieve this goal, we estimate transformation matrices ${ }_{C 1}^{W} M$ and ${ }_{C 2}^{W} M$ from sensors coordinate systems to world coordinate system. The point cloud alignment problem involves finding the optimal transformation (rotation $R$ and translation $t$ ) that will transform the points from the sensor frame in the world frame. The transformation matrix has 12 degrees of freedom. These degrees can be expressed as combinations of 3 parameters from 3D translation $\left(t_{x}, t_{y}, t_{z}\right)$ and 9 parameters from the matrix that combines rotation, scaling and shearing. These degrees of freedom can be viewed as the 9 elements of a $3 \times 3$ matrix plus the 3 components of a vector shift. In our case, we have 2 cameras with a fixed distance between them. The transformation preserves the shape and the size. This is in contrast to an affine transform, which includes scaling and shearing. As a result, scaling and shearing are not considered. Therefore, the number of unknown variables in the transformation matrix is 6 variables $\left(\theta_{x}, \theta_{y}, \theta_{z}, t_{x}, t_{y}, t_{z}\right)$. The rotation matrix and the translation vector have three degrees of freedom each, in total 6. By searching the $R-t$ parameter space (6 dimensions, 3 rotations and 3 translations), we can find the transformation given two sets of corresponding $3 \mathrm{D}$ points data.

$$
M=\left[\begin{array}{cc}
R & t \\
000 & 1
\end{array}\right]=\left[\begin{array}{cccc}
r_{11} & r_{12} & r_{13} & t_{x} \\
r_{21} & r_{22} & r_{23} & t_{y} \\
r_{31} & r_{32} & r_{33} & t_{z} \\
0 & 0 & 0 & 1
\end{array}\right]
$$

It has been shown that the minimum number of required points to solve this problem is 3 [13]. However, using small number of corresponding 3D points can lead to large errors of the reconstructed points. More efficient approaches can be used to calculate transformation matrix. These included shape matching which is based on detecting corresponding points in a shape when changing the view angle. A marker containing a shape can be placed in the field of view of the two cameras. This shape is detected by each camera then compared to a known shape. To do this, we used Procrustes analysis, which is the process of superimposing one collection of marker configurations on another by translating and scaling then rotating them so that the distances between corresponding points in each configuration are as small as possible. The used distance is called Procrustes distance [14]. It is based on a least-square fit method (Equation 2) and requires two aligned shapes with one-to-one point correspondence.

$$
P_{d}^{2}=\sum_{j=1}^{n}\left[\left(x_{j 1}-x_{j 2}\right)^{2}+\left(y_{j 1}-y_{j 2}\right)^{2}\right]
$$

In Equation 2, the metric $P_{d}^{2}$ is the squared Procrustes distance between two shapes, $X_{1}=\left(x_{11}, x_{21}, \ldots x_{n 1}\right)^{T}$ and $X_{2}=\left(x_{12}, x_{22}, \ldots x_{n 2}\right)^{T}$. This represents the sum of the squared point distances. Mathematically formulated, we want to find the best rotation and translation $R$ and $t$ in Equation 3, which transforms a detected shape $X_{1}$ by the camera to an already known shape $X_{2}$.

$$
X_{2}=R * X_{1}+t
$$

In Equation 3, $X_{2}$ is the known shape and $X_{1}$ is the detected shape. $R, t$ are the rotation and the translation applied to detected shape $X_{1}$ to align it with the known shape $X_{2}$. This is formulated as a mathematical programming problem minimizing the Procrustes distance to find the optimal rotation and translation. It can be divided into four steps. First, the centroids of both dataset are computed. Second, both dataset are moved to the origin. Third, the optimal rotation (matrix $R$ ) is found and finally, the translation $t$ is deducted from the previous steps.

\section{B. Thoraco-abdominal Region segmentation}

A rectangular ROI is extracted using Cloud Compare [15] with all clouds selected at once and aligned together. The ROI is used to reduce the computation time. The segmented area contains the thoraco-abdominal region. Note that we do not need to segment the ROI accurately, since we will use a subtraction technique to detect the moving volume due to the chest contraction and expansion. Additionally, the cloud is enhanced and cleaned using Statistical Outlier Removal filter of the Point Cloud Library (PCL) [16]. This filter considers the distance to the underlying surface instead of the distance to the neighbors.

\section{Surface reconstruction}

The volume cannot be computed directly from a point cloud because of the presence of holes. For this reason, we need to generate an intermediary mesh, and then close the holes in it. If the mesh presents many holes, the calculated volume may be invalid. Moreover, if the mesh is not totally closed, the volume cannot be calculated. The benefit of using meshes instead of point clouds directly is the fact that it is easier to close all the holes with a closing reconstruction method. Surface reconstruction follows three main steps. First, the surface is scanned and the point cloud is computed from the scan. Given that normals are very important for surface reconstruction and that they are not already provided by the acquisition device, we use minimum spanning tree propagation technique [17] to compute and orient normals. This method is convenient when the surface is open. The idea is to construct a graph over the point cloud for all the vertexes through the $k$-nearest neighbors of each point and get the orientation of the vertex that has the highest $\mathrm{z}$ value. Then, we go across the graph and correct the orientation of all the vertexes. Finally, the surface is reconstructed using Poisson surface reconstruction algorithm [11], 
Sensor 1 coordinate frame

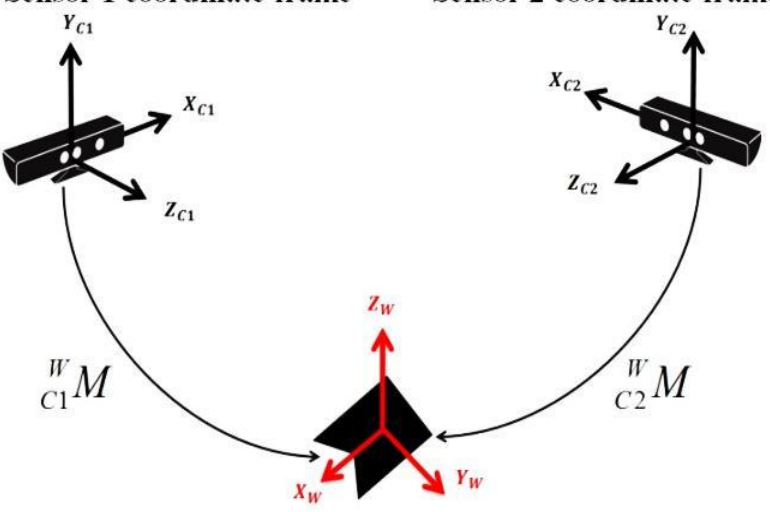

World coordinate frame

Fig. 2. Illustration of transformations between world coordinate frame $\left(X_{w}, Y_{w}, Z_{w}\right)$ and cameras coordinate frames $\left(X_{C i}, Y_{C i}, Z_{C i}\right), i \in\{1,2\}$.

which takes as input a set of points with oriented normals and computes a closed volume. This method is used due to its efficiency in surface reconstruction. It acts on a set of 3D points with oriented normals and solves for an approximate indicator function of the inferred solid, whose gradient best matches the input normals. The indicator function is zero everywhere except near the surface. The object is extracted using "marching cubes algorithm" [18] and the volume is estimated using a subdivision technique by considering a reference plane at a predefined distance from the subject's back and the lateral chest wall.

\section{Volume-time curve estimation}

From the reconstructed surface properly closed, we can simply calculate the volume. One common method to represent a $3 \mathrm{D}$ object is to use octrees, which are hierarchical tree structures. Cloud Compare relies on this structure that enables great performances. An octree partitions a 3-dimensional space by recursively subdividing it into eight sub-cubes. The subdivision is stopped if regions are empty. Otherwise, the occupied spaces are subdivided until reaching a minimal number of points.

The volume of the segmented area is calculated for each frame. The change of this volume is the key to estimating the volume-time curve. It is used as a surrogate data in order to estimate the breathing pattern. Therefore the position of the reference plane is not important. The tidal volume is estimated from the volume-time curve by differencing volumes values between consecutive maximums and minimums in the curve. Figure 3 illustrates how we estimate the volume-time curve. The segmented ROI volume is computed for each frame. Then, volume-time curve is estimated in order to extract Vt and RR.

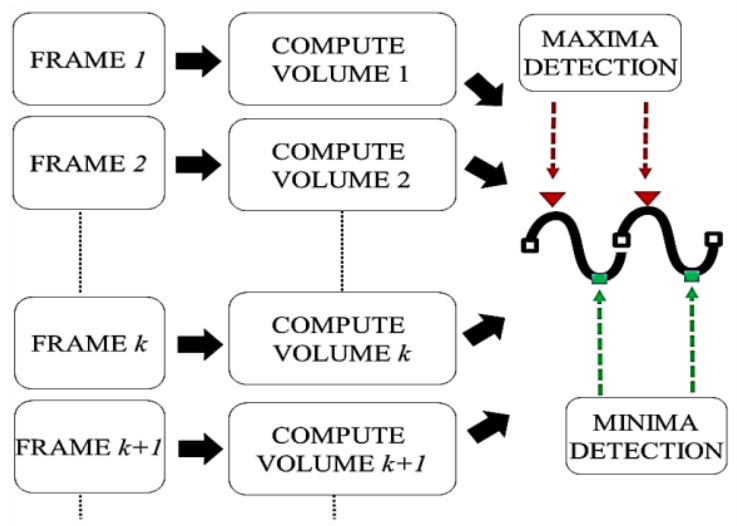

Fig. 3. Volume-time curve estimation.

\section{EXPERIMENTS}

We compared our RR and Vt measurements to the measurements obtained from a mechanical ventilator, considered as a gold-standard widely employed in Pediatric Intensive Care Unit. We performed multiple experiments to measure the most important breathing parameters by analyzing volume changes.

\section{A. Data acquisition}

In our experiments, we used a baby manikin designed according to neonatal anatomical and physiological characteristics. Our experiments are performed in an intensive care room at Sainte-Justine Hospital in Montreal. This environment presents the same challenges experienced by paramedics and physicians in the real world.

The experimental environment includes a manikin, a patient bed with suitable holes to fix the cameras, two RGB-D sensors (Microsoft Kinect V2 [19]) placed on the top and the bottom of the bed, a medical trolley carrying 2 laptops connected to the cameras, and a mechanical ventilator (Servo i, Maquet Inc, Sweden) connected to a lung simulator for infants (MAQUET Medical Systems, 1 Liter Test Lung 190) used as an artificial test lung. The ventilator is a bedside machine. It is attached to the valve of the artificial lung using a breathing tube as shown in Fig. 4. Caregivers can adjust the ventilator according to the baby's condition. Figure 5 illustrates the location of the marker, which we place on the bed in such a way to be in a common field of view of the two cameras. Once the cameras infer their relative positions from the detected marker, we remove it and we place the manikin. Figure 6 shows the sensors locations on the bed. The sensors were positioned in the top right and the bottom left of the bed, both in the direction of $45^{\circ}$ and at a distance of $1 \mathrm{~m}$ to the crib mattress. This positioning offers a high spatial coverage since we cover the top and lateral sides of the baby. We consider the knowledge of lateral surface motion as valuable and offering more complete information for the torso reconstruction.

To evaluate the precision of our method, different volumes were introduced into the artificial test lung. We simulate spontaneous breathing of a child with the ventilator associated to the artificial lung in order to compute $\mathrm{Vt}$ and $\mathrm{RR}$ and perform a comparison with the ventilator reference values.

Two primary modes are used to push the air into the artificial lungs: the neonatal and the adult mode. The air volumes for neonatal mode are respectively: $10 \mathrm{ml}, 20 \mathrm{ml}, 30 \mathrm{ml}, 40 \mathrm{ml}, 50 \mathrm{ml}$ and $100 \mathrm{ml}$. For adult mode, the volumes are respectively: $150 \mathrm{ml}, 200 \mathrm{ml}, 250 \mathrm{ml}$, $300 \mathrm{ml}, 350 \mathrm{ml}, 400 \mathrm{ml}, 450 \mathrm{ml}$ and $500 \mathrm{ml}$. The complete settings are listed in Table 1.

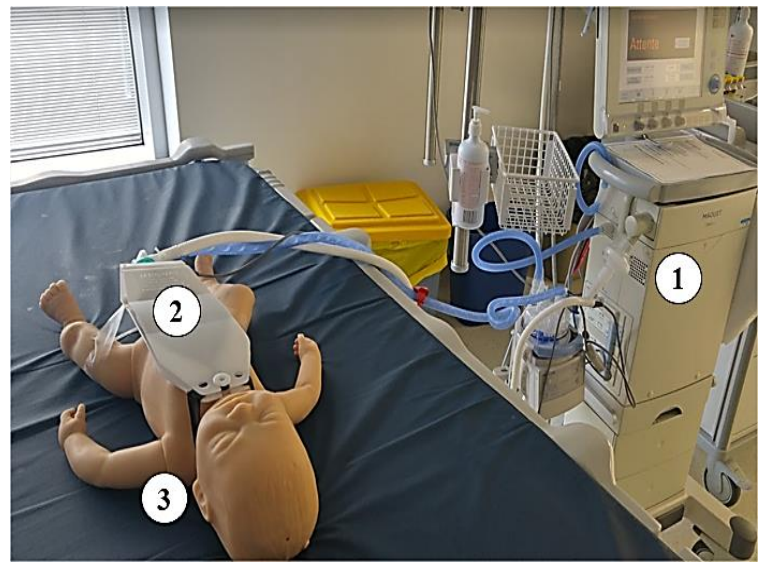

Fig. 4. Experimental materials: (1) Ventilator, (2) Test lung, (3) Baby manikin. 


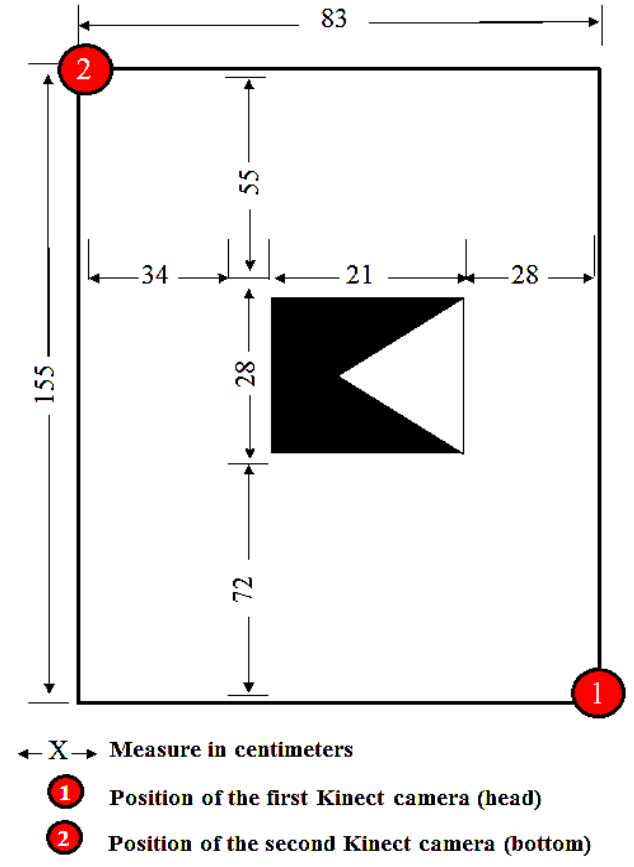

Fig. 5. Bed dimensions and marker location (measures in $\mathrm{cm}$ ).

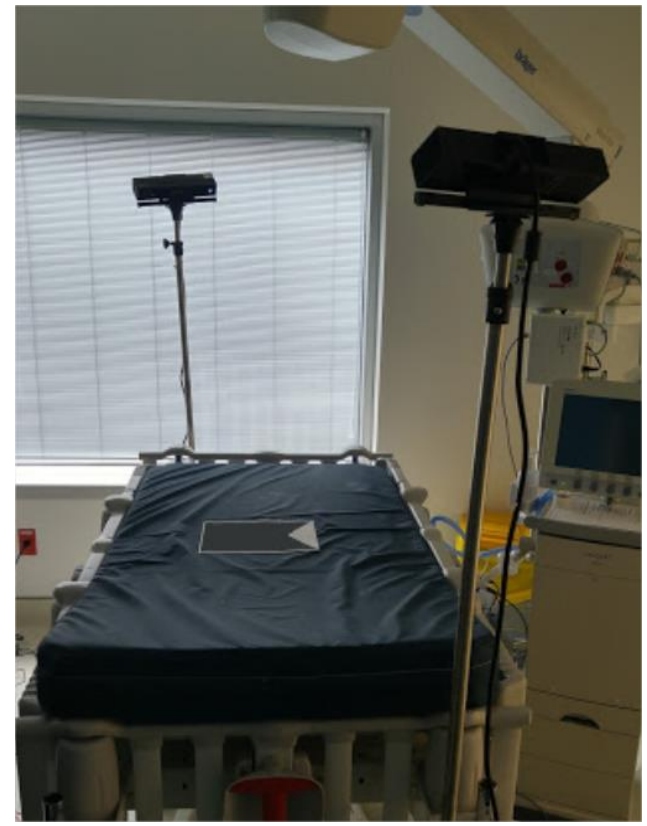

Fig. 6. Experimental set-up and Kinect sensors locations during the experiment.

The experiments were performed under rigorous clinical conditions to avoid interfering with patient care and disturbing caregivers. Our system allows a fast collection of data in the intensive care specific environment. $\mathrm{Vt}$ and $\mathrm{RR}$ were recorded by the mechanical ventilator simultaneously with the cameras recordings. Each camera is connected to a laptop running the data acquisition software. The latter was developed using Visual Studio 2015, Kinect SDK v2. The program stores all RGB, depth images and aligned point clouds to the server (computer 1) with a frame rate of $7 \mathrm{fps}$. Our method for tidal volume calculation uses the point cloud library (PCL) and Cloud Compare software (CC). Point clouds were refined using Autodesk Meshmixer software [20].

\section{B. Respiratory parameter calculation}

The experiments were performed for different ventilator settings corresponding respectively to adult and pediatric modes. For each experiment, we recorded a one minute sequence using our acquisition software. The experimental results are shown in Table 2.

Table 1. Reference ventilator settings with $V t$ : tidal volume, $R R$ : respiratory rate and $I T$ : Inspiration Time.

\begin{tabular}{|l|c|c|c|c|}
\hline & $\begin{array}{c}\text { EXPERIMENT } \\
\text { NUMBER }\end{array}$ & $\begin{array}{c}V t \\
(\mathrm{ml})\end{array}$ & $\begin{array}{c}\text { RR } \\
\text { (resp./min) }\end{array}$ & $\begin{array}{c}\text { IT } \\
\text { (seconds) }\end{array}$ \\
\hline Adult & 1 & 500 & 20 & 0.9 \\
mode & 2 & 450 & 20 & 0.9 \\
& 3 & 400 & 20 & 0.9 \\
& 4 & 350 & 20 & 0.9 \\
& 5 & 300 & 20 & 0.9 \\
& 6 & 250 & 20 & 0.9 \\
& 7 & 200 & 30 & 0.9 \\
& 8 & 150 & 30 & 0.9 \\
Pediatric & 9 & 100 & 30 & 0.9 \\
mode & 10 & 50 & 40 & 0.5 \\
& 12 & 40 & 40 & 0.5 \\
& 13 & 30 & 40 & 0.5 \\
& 14 & 20 & 40 & 0.5 \\
\hline
\end{tabular}

In Fig. 7, the mechanical ventilator settings were put in the adult mode. We then switch the ventilator settings to the neonatal and pediatric mode in order to test our method with small volumes. The volume curves correspond to the first 13 seconds representing approximately 40 frames.

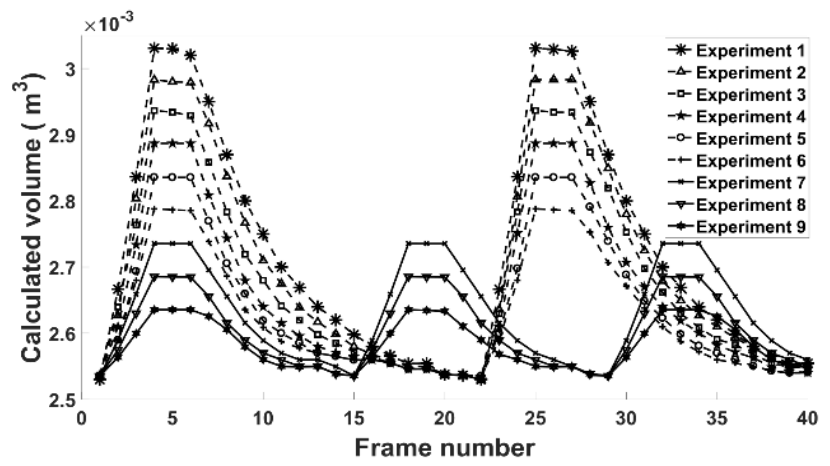

Fig. 7. Adult mode volume-Time curves obtained from our imaging method. The corresponding experiment settings are listed in Table 1.

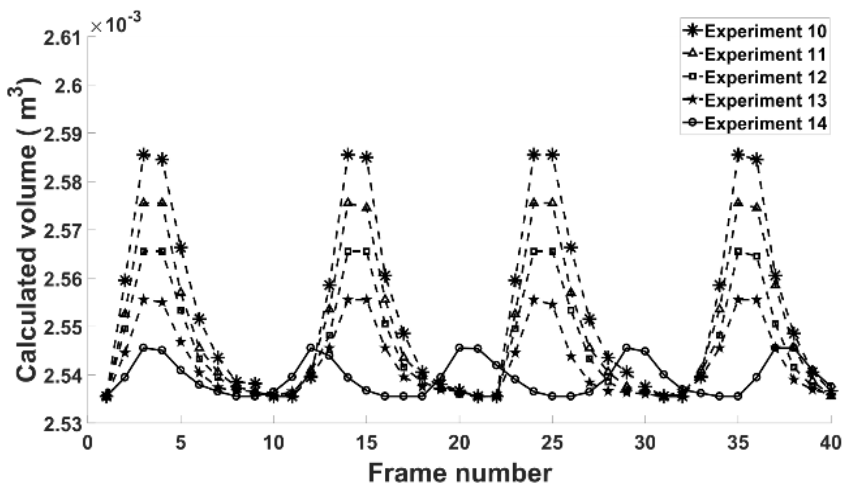

Fig. 8. Neonatal mode volume-Time curves obtained from our imaging method. 
Table 2. Reference ventilator values and our imaging method results with $V t$ : tidal volume, $R R$ : respiratory rate and IT: Inspiration Time. The second value in the columns showing our method results, represents the standard deviation of the result over one minute.

\begin{tabular}{|c|c|c|c|c|c|c|c|}
\hline & \multirow{2}{*}{$\begin{array}{c}\text { EXPERIMENT } \\
\text { NUMBER }\end{array}$} & \multicolumn{2}{|c|}{$\mathrm{Vt}(\mathrm{ml})$} & \multicolumn{2}{|c|}{ RR (resp./min) } & \multicolumn{2}{|c|}{ IT (seconds) } \\
\hline & & Ventilator & Our method & Ventilator & Our method & Ventilator & Our method \\
\hline \multirow{9}{*}{$\begin{array}{l}\text { Adult } \\
\text { mode }\end{array}$} & 1 & 500 & $498.64 \pm 3.93$ & 20 & $19.1973 \pm 1.09$ & 0.9 & $0.9493 \pm 0.13$ \\
\hline & 2 & 450 & $453.36 \pm 1.27$ & 20 & $19.8329 \pm 0.87$ & 0.9 & $0.9454 \pm 0.11$ \\
\hline & 3 & 400 & $401.47 \pm 2.85$ & 20 & $18.9734 \pm 1.03$ & 0.9 & $0.9756 \pm 0.19$ \\
\hline & 4 & 350 & $348.53 \pm 2.49$ & 20 & $19.3495 \pm 0.34$ & 0.9 & $0.9463 \pm 0.24$ \\
\hline & 5 & 300 & $298.91 \pm 2.66$ & 20 & $20.4946 \pm 0.94$ & 0.9 & $0.9317 \pm 0.15$ \\
\hline & 6 & 250 & $250.09 \pm 3.35$ & 20 & $19.9994 \pm 0.25$ & 0.9 & $0.9983 \pm 0.18$ \\
\hline & 7 & 200 & $200.37 \pm 1.93$ & 30 & $29.3494 \pm 0.97$ & 0.9 & $0.9195 \pm 0.11$ \\
\hline & 8 & 150 & $149.63 \pm 3.25$ & 30 & $29.2494 \pm 0.96$ & 0.9 & $0.9156 \pm 0.09$ \\
\hline & 9 & 100 & $101.37 \pm 3.18$ & 30 & $29.4945 \pm 1.02$ & 0.9 & $0.9865 \pm 0.12$ \\
\hline \multirow{5}{*}{$\begin{array}{l}\text { Pediatric } \\
\text { mode }\end{array}$} & 10 & 50 & $48.63 \pm 2.96$ & 40 & $39.9548 \pm 0.94$ & 0.5 & $0.4851 \pm 0.18$ \\
\hline & 11 & 40 & $44.37 \pm 3.25$ & 40 & $39.3861 \pm 1.01$ & 0.5 & $0.4946 \pm 0.08$ \\
\hline & 12 & 30 & $27.63 \pm 2.50$ & 40 & $39.3804 \pm 0.88$ & 0.5 & $0.4995 \pm 0.12$ \\
\hline & 13 & 20 & $21.05 \pm 2.19$ & 40 & $39.6108 \pm 0.67$ & 0.5 & $0.5008 \pm 0.13$ \\
\hline & 14 & 10 & $7.95 \pm 2.88$ & 50 & $49.4554 \pm 0.79$ & 0.5 & $0.5204 \pm 0.11$ \\
\hline
\end{tabular}

1) Respiratory rate calculation: We compute the average duration of one respiratory cycle $(D)$ by averaging distances between consecutive peaks. In Equation 4, we calculate $D$ by summing the distances between peaks and dividing this sum by the number of peaks in one minute. Equation 5 presents how we calculate the standard deviation, which provides an estimate of the variability of $D$. RR is deducted from $D$ using Equation 6, in which we use the same unit given by the ventilator (respirations/minute).

$$
\begin{aligned}
& D=\frac{\sum_{i=1}^{N_{\text {peaks }}{ }^{-1}} d_{i}}{N_{\text {peaks }}-1} \\
& \Sigma_{D}=\sqrt{\frac{\sum_{i=1}^{N_{\text {peaks }}-1}\left(d_{i}-D\right)^{2}}{N_{\text {peaks }}-1}}
\end{aligned}
$$

where $N_{p}$ represents the number of peaks of the volume-time curve in a minute, $d_{i}$ is the distance between peaks $i$ and $i+1, D$ is the average duration of a respiratory cycle, and $\Sigma_{D}$ represents the standard deviation of $D$.

$$
R R(\text { respirations } / \text { minute })=\frac{60 \text { seconds }}{D}
$$

2) Tidal volume calculation: Tidal volume per cycle is the volume of air inhaled or exhaled from a person's lungs in a cycle. This definition is true unless we have air leak during mechanical ventilation. Indeed, tidal volume in a cycle can be calculated as the average value of inspiratory and expiratory volumes, while tidal volume per minute is the average of all tidal volumes during a minute. It can be calculated using Equation 7:

$$
V t=\frac{\sum_{i=1}^{N p} t v_{i}}{N_{p}}
$$

where $V t$ is the average tidal volume over one minute and $t v_{i}$ is the tidal volume of the cycle $i$. The standard deviation provides an estimate of the tidal volume variability in all the respiratory cycles using Equation 8:

$$
\Sigma_{V t}=\sqrt{\frac{\sum_{i=1}^{N_{p}}\left(t v_{i}-V t\right)^{2}}{N_{p}}}
$$

where $\Sigma_{T V}$ represents the standard deviation of the tidal volume
3) Inspiratory time calculation: The inspiratory time is the amount of time taken to deliver the tidal volume of air to the lung. It can be extracted from the volume-time curve. The Average inspiratory time per minute can be calculated as the average of all extracted inspiratory times during a minute (Equation 9).

$$
T I=\frac{\sum_{i=1}^{N_{p}} t i_{i}}{N_{p}}
$$

where $T I$ is the average inspiratory time over one minute and $t i_{i}$ is the inspiratory time of the cycle $i$. The variability of the inspiratory time over one minute is measured using Equation 10.

$$
\Sigma_{T I}=\sqrt{\frac{\sum_{i=1}^{N p}\left(t i_{i}-T I\right)^{2}}{N_{p}}}
$$

where $\Sigma_{T I}$ represents the standard deviation of the inspiratory time over $N_{p}$ samples.

\section{Experimental results}

All the experiments have been performed for the duration of one minute. The results are shown in Table 2 and were compared to the reference ventilator values.

The resulting root mean square deviation (RMSD) between the measured and the reference values shows an error of $\pm 1.94 \mathrm{ml}, \pm 0.59$ respirations per minute and $0.05 \mathrm{~s}$ for respectively, tidal volume, respiratory rate and inspiratory time. A small RMSD, which is a good measure of accuracy, indicates that the estimated values by our method are very close to the reference values given by the ventilator. The differences of $\mathrm{Vt}$ mean values given by the ventilator and our method are in the scale of a few milliliters while maximum difference between the 2 methods does not exceed $4.37 \mathrm{ml}$ (in experiment 11). Also, the differences of RR mean values given by the 2 methods are in the order of one respiration per minute. Similarly, for mean IT values, the differences between the 2 methods lies in the range of 0.008 seconds and 0.0983 seconds. We can observe that our method works a better for big volumes than small volumes. The results are very accurate for the volumes: $200 \mathrm{ml}$ and $250 \mathrm{ml}$. They are less accurate for very small volumes, such as for $10 \mathrm{ml}$ volume. For this volume, the estimated value was $7.95 \mathrm{ml}$ and can decrease until $5.07 \mathrm{ml}$ (almost half of the real value). Generally, the global results yielded high accuracy and proved that the performance of the designed system was comparable to existing respiratory evaluation devices. 


\section{CONCLUSION}

This paper presented a vision-based method for respiratory volume estimation without any physical contact. Our system first computes point clouds and aligns them in a common coordinate system. The point cloud is subsequently segmented and a $3 \mathrm{D}$ model is computed by considering a reference plane at a predefined distance from the subject's back and lateral chest wall. The volume can be then easily computed based on an octree subdivision technique of the 3D space. Finally respiratory parameters are calculated from the estimated volume-time curve. The system is a promising support tool designed to assist caregivers. The experimental results have shown the robustness and the effectiveness of our approach. Hence, the system is able to accurately track torso volume changes. It has also the advantages of low-cost and ease of setup. For future work, we aim to design fully integrated and straightforward software based on this work. This includes the development of a robust GPU-based platform for real-time acquisition, processing and data analysis for a more practical level. Furthermore, we will focus on characterizing respiratory distress, particularly the retraction signs of the breast (RSB).

\section{ACKNOWLEDGMENT}

This work was supported by grants from the Natural Science and Engineering Research Council of Canada (NSERC) and Medteq.

\section{REFERENCES}

[1] S. Essouri, F. Baudin, L. Chevret, M. Vincent, G. Emeriaud and P. Jouvet. Variability of Care in Infants with Severe Bronchiolitis: Less-Invasive Respiratory Management Leads to Similar Outcomes. The Journal of Pediatrics, 2017.

[2] B. Ying-Wen, L. Wen-Tai and C. You-Wei. Design and implementation of an embedded monitor system for detection of a patient's breath by double Webcams. Medical Measurements and Applications Proceedings (MeMeA), 2010 IEEE International Workshop on. IEEE, 2010.

[3] J. Xia and R. Siochi. A real-time respiratory motion monitoring system using KINECT: Proof of concept. Medical physics, 39(5): 2682-2685, 2012.

[4] F. Tahavori, M. Alnowami, and K. Wells, Marker-less Respiratory Motion Modeling Using the Microsoft Kinect for Windows, in SPIE Medical Imaging, 1-10, 2014.

[5] M.C. Yu, H. Wu; J.L. Liou; M.S. Lee; Y.P Hung. Breath and Position Monitoring during Sleeping with a Depth Camera. In Proceedings of the International Conference on Health Informatics, Algarve, Portugal, 1-4 February 2012.

[6] H. Aoki, H. Nakamura; K. Fumoto, K. Nakahara, M. Teraoka. Basic study on non-contact respiration measurement during exercise tolerance test by using kinect sensor. In Proceedings of the 2015 IEEE/SICE International Symposium on System Integration (SII), Nagoya, Japan, 11-13 December 2015.

[7] P. Henry, M. Krainin, E. Herbst, X. Ren and D. Fox. RGB-D mapping: Using Kinect-style depth cameras for dense 3D modeling of indoor environments. The International Journal of Robotics Research, 31(5): 647663, 2012.

[8] K. Khoshelham. Accuracy analysis of kinect depth data. In ISPRS workshop laser scanning,38,(5):12, August 2011.

[9] K. Khoshelham and S.O. Elberink. Accuracy and resolution of kinect depth data for indoor mapping applications. Sensors, 12(2): 1437-1454, 2012.

[10] E. Lachat, H. Macher, M.A. Mittet, T. Landes and P. Grussenmeyer. First experiences with Kinect v2 sensor for close range 3D modelling. The International Archives of Photogrammetry, Remote Sensing and Spatial Information Sciences, 40(5): 93, 2015.

[11] M. Kazhdan and H. Hoppe. Screened poisson surface reconstruction. ACM Transactions on Graphics (TOG), 32(3): 29, 2013.

[12] A. Hornung, K.M. Wurm, M. Bennewitz, C. Stachniss and W. Burgard. OctoMap: An efficient probabilistic 3D mapping framework based on octrees. Autonomous Robots, 34(3): 189-206, 2013.

[13] K. Grauman and B. Leibe. Visual object recognition. Synthesis lectures on artificial intelligence and machine learning, 5(2): 1-181, 2011.

[14] F.J. Rohlf. Shape statistics: Procrustes superimpositions and tangent spaces. Journal of Classification, 16(2): 197-223, 1999.

[15] D. Girardeau-Montaut. Cloud Compare - 3D Point Cloud and Mesh Processing Software. Open Source Project, 2015.
[16] R.B. Rusu and S. Cousins. 3d is here: Point cloud library (pcl). In 2011 IEEE International Conference on Robotics and Automation, May 1-4, 2011.

[17] S. Pettie and V. Ramachandran. An optimal minimum spanning tree algorithm. In Journal of the ACM (JACM), 49(1): 16-34, 2002.

[18] W.E. Lorensen and H. E. Cline. Marching cubes: A high resolution 3D surface construction algorithm. In ACM siggraph computer graphics, 21( 4): 163-169, 1987.

[19] P. Fankhauser, M. Bloesch, D. Rodriguez, R. Kaestner, M. Hutter and R. Siegwart. Kinect v2 for mobile robot navigation: Evaluation and modeling. In 17th International Conference on Advanced Robotics (ICAR), July 2015

[20] Autodesk ${ }^{\circledR}:$ Meshmixer ${ }^{\mathrm{TM}}$. http://www.meshmixer.com/, 2017. [accessed 01-06-2017]. 